\title{
EXPRESSION OF ERG IMMUNOHISTOCHEMICAL STAIN IN PROSTATIC ADENOCARCINOMA
}

\author{
Sana Tabish, Erum Khan, Sajid Mushtaq, Usman Hassan, Mudassar Hussain \\ Shaukat Khanum Memorial Cancer Hospital \& Research Centre, Lahore Pakistan
}

\begin{abstract}
Objective: To investigate the incidence ERG fusion positive adenocarcinoma and its correlation with gleason grade and gleason group.

Study Design: Cross-sectional study.

Place and Duration of Study: Shaukat Khanum Memorial Cancer Hospital \& Research Centre (SKMCH \& RC), Lahore Pakistan, from Jan 2018 to Jan 2019.

Methodology: A total of 100 cases of prostatic adenocarcinoma were retrieved and ERG immunohistochemical stain was applied on all these cases. ERG expression, extent of staining, percentage of tumor cells positive for ERG, pattern of staining was recorded. We found out relationship of ERG expression and gleason grade/gleason group.

Results: ERG expression was seen in 62/100 (62\%) cases. Mean patient age of ERG positive prostatic adenocarcinoma patients was 69.68 years. In GG1 (14.5\%) 9 cases exhibited ERG positivity. In GG2-3, 41.93\% cases and in GG4-5, 43.54\% cases showed ERG expression.

Conclusion: To conclude, age of presentation of ERG positive adenocarcinoma was high. ERG expression was seen in $62 \%$ of our cases which was much higher than reported in other Asian countries. In our study we found a significant correlation between ERG expression and high gleason grade group.
\end{abstract}

Keywords: ERG expression, Gleason grade group, Prostatic adenocarcinoma.

This is an Open Access article distributed under the terms of the Creative Commons Attribution License (https://creativecommons.org/licenses/by-nc/4.0/), which permits unrestricted use, distribution, and reproduction in any medium, provided the original work is properly cited.

\section{INTRODUCTION}

Prostatic cancer is second most frequently diagnosed malignancy worldwide ${ }^{1}$. This increased incidence of prostatic cancer (PCa) is due to early detection of low volume tumor by screening with prostate specific antigen ${ }^{1}$. Wide range of variations in incidence and mortality rates have been observed amongst different populations. Incidence of prostatic cancer in Pakistan is $5.6 \% 1$.

Prostatic adenocarcinoma shows marked molecular heterogeneity. Most prevalent molecular alteration is TMPRSS2: ERG leading to over expression of ERG oncoprotein $^{2,3}$. Prevalence of TMPRSS2: ERG fusion PCa show wide geographic variation. Epidemiological data has shown increased incidence in western countries $^{1}$. This gene fusion is an early event in prostatic carcinoma development with positive expression in precursor lesion 4 .

Several publications document incidence of ERG over expression and its association with age, morphological features and prognostically important pathological parameters ${ }^{5-9}$. ERG fusion positive PCa has predominantly been reported in young patients ${ }^{5-7}$. Fusion

Correspondence: Dr Sana Ghulam Nabi, Pathology Dept, Shaukat Khanum Memorial Cancer Hospital \& Research Centre, Lahore Pakistan Received: 23 Oct 2020; revised received: 22 Jan 2021; accepted: 25 Jun 2021 positive PCa is associated with low PSA level as compared to fusion negative $\mathrm{PCa}^{7,8}$.

Cribriform growth, blue tinged mucin and macronucleoliare confirmed morphological features present in fusion positive $\mathrm{PCa}^{8}$. Numerous studies have evaluated the correlation between ERG expression and pathological grade. Contrasting results are available. Some studies show association with high pathological grade other show an inverse correlation 2,3,7,9. There are no substantial associations between ERG expression and pathological grade according to some studies ${ }^{8}$. High grade prostatic intraepithelial neoplasia (HGPIN) is a knownprecursor lesion for prostatic adenocarcinoma. High grade prostatic intraepithelial neoplasia adjacent to fusion positive prostatic adenocarcinoma also show expression for $\mathrm{ERG}^{3,10}$. Prostatic carcinoma is best known for molecular heterogeneity and multifocality. PCa in some patients harbor both fusion positive and fusion negative tumor 2,9 . Dismal prognosis along upsurge risk of biochemical recurrence and the cancer specific death has been reported in non-surgically treated fusion positive $\mathrm{PCa}^{6,7}$.

Fluorescent in situ hybridization (FISH) and immunohistochemistry (IHC) are used for the detection of ERG gene fusion. Highly concordant results have been reported between fluorescent in situ hybridization and ERG immunohistochemical stain with $96.5 \%$ specificity 
and $95.7 \%$ sensitivity ${ }^{9,10,12}$. ERG immunohistochemical stain is an important surrogate marker of TMPRSS2: ERG gene fusion with negative expression in benign glands.

In this study, we observed the expressions of ERG and its correlation to gleason score and gleason levels in prostatic adenocarcinoma.

\section{METHODOLOGY}

It was a cross-sectional study conduc-ted at Histopathology department of Shaukat Khanum Cancer Memorial Hospital and Research Centre (SKMCH \& RC) Lahore. After ethical approval from the SKMCH \& RC Review Board (EX-06-08-19-02) 100 cases of prostatic adenocarcinoma including all Gleason grades were obtained from archives of SKMCH \& RC, from January 2018 to January 2019. Cases with poor fixation were excluded from the study.

All H\&E slides were reviewed and diagnoses were confirmed. For each case we recorded patient age, gleason grade, gleason score, tumor volume and perineural invasion. Most representative slide from each case was selected to perform IHC.

Four to 5 micron thick sections were obtained from parafin embedded block. Epitope retrieval was done by using standard heat-induced method. The primary antibody was incubated for 15 minutes. The clone used for ERG was ERG (DAKO)-rabbit monoclonal (IR659; DAKO, Denmark). Staining was performed on Leica Bond III auto-stainer (Leica, Buffalo Grioe, IL) with a polymer detection kit (Refine, Leica).

Intensity of immunohistochemical stain was interpreted as weak $(1+)$, moderate $(2+)$ and strong $(3+)$. Extent of staining, percentage of tumor cells positive for ERG, pattern of staining (uniform or heterogeneous) was also recorded. Cases with reduced nuclear staining were scored $(1+)$ and cells with no nuclear or cytoplasmic stain were scored $(0)^{11-13}$. Cases with weak $(1+)$ nuclear staining in $<10 \%$ with tumor cells were considered negative, while cases with heavy nuclear staining were considered positive in $<10 \%$ of tumor cells. Negative nuclear staining and cytoplasmic staining were also considered negative. Visual estimation for percentage of tumor cells showing nuclear staining was used. Extent of staining was estimated as more than $75 \%, 50-75 \%, 10-50 \%$ and $<10 \%$.

We analyzed the data by using SPSS-21. Fisherexact test was used to test relationship between ERG staining and gleason grade/gleason group.

\section{RESULTS}

Total 100 cases were enrolled in our study including core biopsy (33 cases, 33\%), transvesical prostatectomy (9 cases, $9 \%$ ) and transurethral resection of prostate (58 cases, $58 \%$ ).

Age range was 45-91 years. In ERG positive prostatic carcinoma minimum age of presentation was 53 years with age range 53-91 years (mean age 69.68 years, median age 69.5 years).

Positive control for ERG (strong nuclear staining in endothelial cells and moderate to weak staining in lymphocytes) was present in all cases. ERG expression was seen in 62 cases $(62 \%)$. Amongst these cases strong staining (3+) was seen in 50 cases $(50 \%)$ and moderate staining $(2+)$ was seen in 12 cases $(12 \%)$ fig- 1 . Negative ERG expression was observed in 38 (38\%) cases fig- 2 .

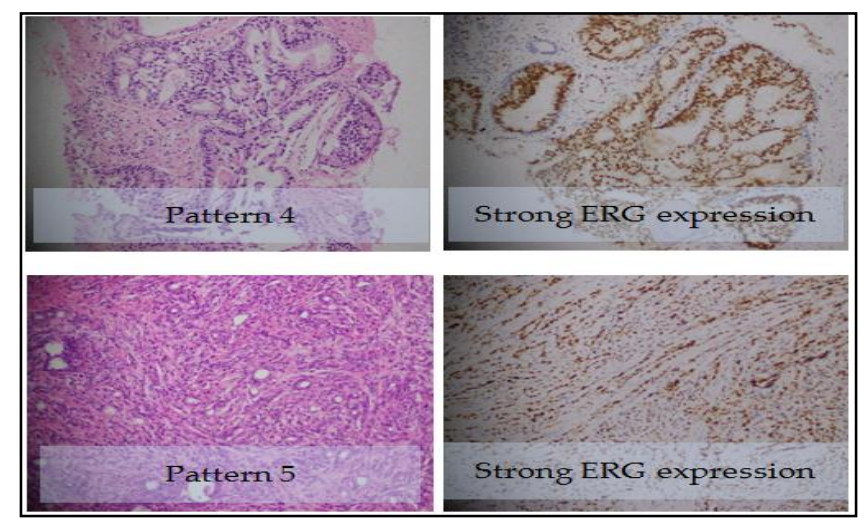

Figure-1: Prostatic adenocarcinoma with strong ERG expression.

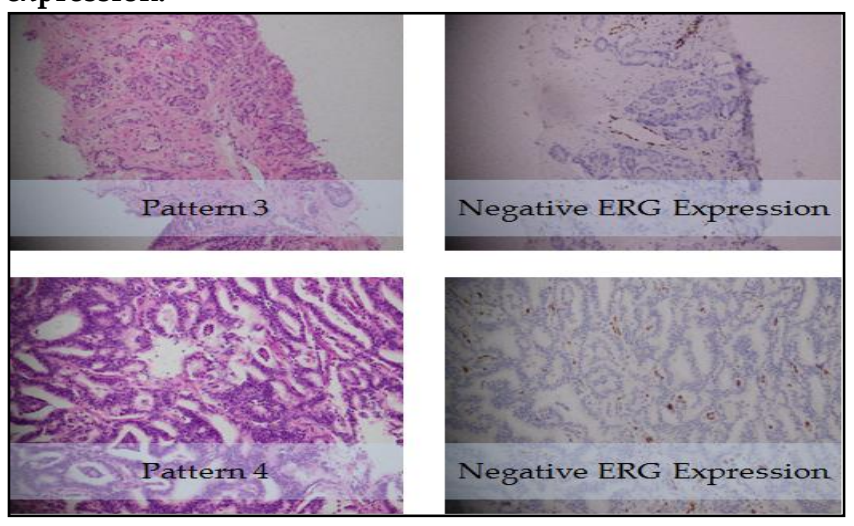

Figure-2: Prostatic adenocarcinoma with negative ERG expression (positive internal control).

In our study total 21 (21\%) cases were diagnosed with prostatic adenocarcinoma gleason group $1(3+3)$. Nine (42\%) out of 21 cases were positive for ERG immunostain. Uniform staining was observed in 7/9 cases $(77.77 \%)$, whereas $2 / 9$ cases $(22.22 \%)$ showed hetero- 
geneous staining pattern. Seven cases $(77.77 \%)$ exhibited ERG expression in $>75 \%$ tumor cells. In one case $(11.11 \%)$ staining was present in $75-50 \%$ of tumor cells. Focal strong staining in $<10 \%$ tumor cells was observed in one $(11.11 \%)$.

Cases with gleason group 2, score 7(3+4) were 16 $(16 \%)$ in number. ERG staining was seen $12 / 16$ (75\%) cases with heterogeneous staining pattern in $9 / 12$ $(75 \%)$ cases and uniform staining in 3/12 (25\%) cases. One case $(8.33 \%)$ with heterogeneous staining pattern showed focal strong staining in pattern 3 and pattern 4 was entirely negative. Eleven $(91.66 \%)$ out of 12 cases demonstrated staining in more $75 \%$ of tumor cells and remaining one $(8.33 \%$ ) case showed staining $<75 \%$ (50$75 \%)$ tumor cells.

Table: Correlation of ERG expression with gleason grade group.

\begin{tabular}{l|c|c|c}
\hline $\begin{array}{l}\text { Gleason } \\
\text { Grade Group }\end{array}$ & $\begin{array}{c}\text { Negative ERG } \\
\text { Expression }\end{array}$ & $\begin{array}{c}\text { Positive ERG } \\
\text { Expression }\end{array}$ & Total \\
\hline GG1 (3+3) & $12(19 \%)$ & $9(14.51 \%)$ & 21 \\
\hline GG2-3 & 4 & 12 & 16 \\
GG2 (3+4) & 5 & 14 & 19 \\
GG3 (4+3) & $9(14 \%)$ & $26(41.93 \%)$ & \\
Total Cases & & & \\
\hline GG4-5 & 1 & 9 & 17 \\
GG4 (4+4) & 0 & 2 & 1 \\
GG4 (3+5) & 4 & 10 & 2 \\
GG4 (5+3) & 4 & 6 & 14 \\
GG5 (4+5) & 1 & 0 & 10 \\
GG5 (5+4) & $18(29.03 \%)$ & $27(43.54 \%)$ & 1 \\
GG5 (5+5) & & & \\
Total Cases & & & \\
\hline
\end{tabular}

Prostatic carcinoma with gleason group 3, gleason score $7(4+3)$ was diagnosed in $19(19 \%)$ patients. ERG expression was seen in $14 / 19(73.68 \%)$ cases with uniform staining pattern in $7(50 \%)$ cases. Heterogeneous staining was observed in $7(50 \%)$ cases. Majority 10 $(66.66 \%)$ showed ERG expressions in $>75 \%$ of tumor cells. Staining in $<75 \%(75-50 \%)$ tumor cells was present in $2(14.28 \%)$ cases,only single case $(7.14 \%)$ showed staining in $<50 \%(10-50 \%)$ tumor cells and in one case $(7.14 \%)$, however, $<10 \%$ tumor cells showed the focal strong staining.

Total 19 cases were diagnosed with gleason group 4. Gleason score $4+4$ was documented in $17 / 19$ $(89.47 \%)$ cases. Gleason score $3+5$ was seen in $1(5.26 \%)$ and $5+3$ in $2(10.52 \%)$ cases. Nine $(52.94 \%)$ out of 17 cases with gleason grade $4+4$ demonstrated ERG positivity. Uniform staining pattern was observed in $6 / 9(66.66 \%)$ cases and heterogeneous staining pattern was present in $3 / 9(33.33 \%)$ cases. More than $75 \%$ ERG expression was seen in $7 / 9(77.77 \%)$ cases. staining in $<75 \%(50-75 \%)$ tumor cells was observed in $1(11.11 \%)$ cases. One $(11.11 \%)$ case showed focal $(<10 \%)$ strong staining in tumor cells. Single case with gleason score $3+5$ showed negative ERG expression. Two (10.52\%) diagnosed case of gleason score $5+3$ were positive for ERG and both casesshowed heterogeneous pattern of staining. In one strong ERG expression was seen in pattern $5(90 \%)$ and pattern 3 showed moderate staining. In second case strong and moderate intensity staining involved both patterns.

Total $25(25 \%)$ cases were diagnosed with gleason group 5. Prostatic carcinoma with gleason score $4+5$ was present in $14 / 25(56 \%)$ cases, gleason score $5+4$ was seen in 10/25 (40\%) and gleason score $5+5$ in $1 /$ $25(4 \%)$ case only. ERG positivity exhibited by $10 / 14$ $(71.43 \%)$ cases with gleason grade $4+5$. Uniform pattern of staining was seen in $7 / 10(70 \%)$ cases and heterogeneous staining pattern was present in 3/10 (30\%) cases.six $(60 \%)$ out of 10 cases showed ERG expression in $>75 \%$ tumor cells. Three $(30 \%)$ out of 10 cases showed expression in $<75 \%$ (75-50\%) tumor cells and one $(10 \%)$ case showed staining in $<50 \%(10-50 \%)$ tumor cells. Six $(60 \%)$ out of 10 cases with gleason pattern $5+4$ showed positive ERG expression. Two (33.33\%) cases showed heterogeneous staining pattern and remaining $4(66.66 \%)$ cases showed uniform pattern of staining. Four $(66.66 \%)$ cases demonstrated ERG expression in $>75 \%$ tumor cells. One $(6 \%)$ case showed focal strong staining in $<10 \%$ tumor cells and in $1(6 \%)$ case staining was seen in $50-75 \%$ of tumor cells. Gleason score $5+5$ was seen in one case only with negative ERG expression.

We grouped our patients into 3 groups as Gleason grade group 1 (GG1), gleason grade group 2-3 (GG2-3), gleason grade group 4-5 (GG4-5) and association of ERG expression was evaluated table. In GG1 (14.5\%) 9 cases exhibited ERG positivity. In GG2-3, 41.93\% cases and in GG4-5, 43.54\% cases showed ERG exp-ression. An increasing trend was observed.

Non neoplastic glands (benign glands and glands with partial and complete atrophy) were present in 80 $(80 \%)$ cases. In $2 / 80(2.5 \%)$ cases benign non atrophic glands were focally positive for ERG and remaining $78(97.5 \%)$ cases were negative. Different patterns of atrophy (complete and partial atrophy) were completely negative.

\section{DISCUSSION}

Prostatic adenocarcinoma is second most common malignancy worldwide particularly in European 
countries. Increasing trends of incidence has now observed in Asian countries due to life style modification ${ }^{1}$. Marked molecular heterogeneity has been documented in prostatic adenocarcinoma ${ }^{2,12}$. Most frequent molecular alteration seen in prostatic adenocarcinoma is TMPRSS2-ERG which can be detected by FISH and ERG immunohistochemical stain ${ }^{2,3}$. Variable data is available regarding diagnostic and prognostic significance of ERG fusion. ERG fusion positive PCa has associated with dismal prognosis with increase chances of biochemical recurrence, metastatic potential and cancer related death $6,7,13$. There are reports showing better response of ERG fusion positive PCa to androgen deprivation therapy, help in stratifying patients for clinical management ${ }^{6}$. ERG re-arrangement can be detected by FISH and IHC. High concordance has been reported between FISH and IHC with $96.5 \%$ specificity and $95.7 \%$ sensitivity ${ }^{10,12}$. ERG immunohistochemical stain can be used as a surrogate marker ${ }^{12}$.

In this study mean patient age in ERG positive PCa was 69.68 years. Young age of presentation in ERG positive PCa was reported in several studies 7 . Mean age of 60 years was stated by Schaefer et al, and Brooks et al. A research conducted by Hashmi et al, documented mean age of 69 years in fusion positive $\mathrm{PCa}^{14}$. Increase age at presentation in our study might be due to late presentation and lack of PSA screening in our population.

Data regarding incidence of ERG positive PCa showed wide variation. Increased prevalence was observed in western countries (54\%) as compared to Asian countries $(23 \%)^{2}$. Study conducted by Abdelsalam et al, demonstrated $42.7 \%$ ERG positive prostate tumor in their cohort ${ }^{15}$. Forty nine percent incidence was documented by verdu et al ${ }^{16}$. Current study ERG expression was observed in $62 \%$ cases which was significantly higher than other Asian countries and almost equivalent to western countries. This wide variation from the previously published data might be due to life style changes and geographical variation even in Asian countries as low incidence was reported in China, Japan, Korea and India ${ }^{1}$.

ERG expression and its correlation with gleason grade group was assessed in many studies and showed contrasting results. Biasmar et al, and Furustao et $a l$, stated association of ERG expression with high gleason score ${ }^{6,12}$. Study conducted by Dubovenko et al, and abdelsalam showed increased expression of ERG in tumors with low gleason score ${ }^{15,17}$. According to Verdu et al, and Mosquera et al, no correlation exist between
ERG expression and gleason score 8.16. In our study no significant correlation found between gleason score and individual grade groups with ERG expression. Significant correlation was found when assessment was done as GG1 (14.5\%), GG2-3 (41.93\%), GG4-5 $(43.54 \%)$. In our study increase in frequency of ERG expression was in tumors with high grade group. According to Hagglof et al, ERG expression decreases the survival even with gleason score 6 as compared to patients with ERG negative prostatic carcinoma with similar gleason score ${ }^{9}$. According to meta-analysis by Zhou ERG fusion was associated with more aggressive tumors in Asian and African population comparatively to western population ${ }^{2}$.

Staining pattern was uniform in 32 cases and heterogeneous in 28 cases. According to literature no survival difference was observed in either uniform or heterogeneous pattern of staining .

Prostatic carcinoma showed marked molecular heterogeneity with some tumor foci harboring ERG fusion and other being negative for ERG ${ }^{2,12}$. One case in our study showed focal strong staining in pattern 3 and pattern 4 was entirely negative might representing molecular heterogeneity.

ERG expression is seen in 50-70\% PCa and its positivity strongly suggest an epithelial malignancy. Its expression in benign glands and non-cancerous lesion is very low. In our study only two cases demonstrated focal ERG positivity in benign glands. This finding was concordant with studies of Yaskiv et al, and Furusato et al11,12. Diagnostic panel used for limi-

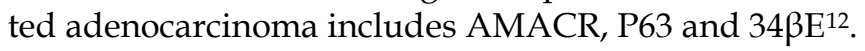
Expression of AMACR in some benign lesion (adenosis and partial atrophy) reducing its sensitivity and specificity. Due to high tumor specific expression, ERG can be used as diagnostic marker in PCa and in tumors of uncertain origin (metastatic workup) ${ }^{18}$. The only limitation of its use as diagnostic marker is its expression in $50-70 \%$ of prostatic adenocarcinoma. ERG immunohistochemical stain in panel with other immunomarkers will be helpful diagnostically. High grade pros-tatic intraepithelial neoplasia adjacent to fusion positive PCa also show expression for ERG, warrant rebiopsy in cases showing only HGPIN with ERG expression. This increases the diagnostic utility of ERG immunostain.

\section{CONCLUSION}

In summary, age of presentation of ERG positive adenocarcinoma was high in our series as compared to previously conducted studies. ERG expression was 
observed in $62 \%$ cases which was much higher than reported in other Asian countries. In our research, the association between ERG and high gleason grade was significant.

\section{CONFLICT OF INTEREST}

This study has no conflict of interest to be declared by any author.

\section{REFERENCES}

1. Kimura T, Egawa S. Epidemiology of prostate cancer in Asian countries. Int J Urol 2018; 25(6): 524-31.

2. Zhou CK, Young D, Yeboah ED, Coburn SB, Tettey Y, Biritwum $\mathrm{RB}$, et al. TMPRSS2: erg gene fusions in prostate cancer of west african men and a meta-analysis of racial differences. Amer J Epidemiol 2017; 186(12): 1352-61.

3. Abou-Ouf $\mathrm{H}$, Zhao L, Bismar TA. ERG expression in prostate cancer: biological relevance and clinical implication. J Cancer Res Clin Oncol 2016; 142(8): 1781-93.

4. Aldaoud N, Abdo N, Al Bashir S, Alqudah M, Marji N, Alzou'bi $\mathrm{H}$, et al. Prostate cancer in Jordanian-Arab population: ERG status and relationship with clinicopathologic characteristics. Virchows Arch 2017; 471(6): 753-59.

5. Aldaoud N, Abdo N, Al Bashir S, Alqudah M, Marji N, Alzou'bi $\mathrm{H}$, et al. Prostate cancer in Jordanian-Arab population: ERG status and relationship with clinicopathologic characteristics. Virchows Arch 2017; 471(6): 753-59.

6. Bismar TA, Hegazy S, Feng Z, Yu D, Donnelly B, Palanisamy N, et al. Clinical utility of assessing PTEN and ERG protein expression in prostate cancer patients: a proposed method for risk stratification. J Cancer Res Clin Oncol 2018; 144(11): 2117-25.

7. Carneiro A, Barbosa ÁRG, Takemura LS, Kayano PP, Moran NKS, Chen CK, et al. The role of immunohistochemical analysis as a tool for the diagnosis, prognostic evaluation and treatment of prostate cancer: a systematic review of the literature. Front Oncol 2018; 8(1): 1-9.

8. Mosquera J, Mehra R, Regan MM, Perner S, Genega EM, Bueti
G, et al. Prevalence of TMPRSS2-ERG fusion prostate cancer among men undergoing prostate biopsy in the United States. Clinical Cancer Res 2009; 15(14): 4706-11.

9. Hägglof C, Hammarsten P, Strömvall K, Egevad L, Josefsson A, Stattin $\mathrm{P}$, et al. TMPRSS2-ERG expression predicts prostate cancer survival and associates with stromal biomarkers. PLoS One 2014; 9(2): e86824-30.

10. Park K, Tomlins SA, Mudaliar KM, Chiu Y, Esgueva R, Mehra $\mathrm{R}$, et al. Antibody-based detection of erg rearrangement-positive prostate cancer. Neoplasia 2010; 12(7): 590-IN21.

11. Yaskiv $O$, Zhang $X$, Simmerman $K$, Daly T, He H, Falzarano S, et al. The utility of ERG/P63 double immunohistochemical staining in the diagnosis of limited cancer in prostate needle biopsies. Amer J Surg Pathol 2011; 35(7): 1062-68.

12. Furusato B, Tan S, Young D, Dobi A, Sun C, Mohamed AA, et al. ERG oncoprotein expression in prostate cancer: clonal progression of ERG-positive tumor cells and potential for ERG-based stratification. Prostate Cancer Prostatic Dis 2010; 13(3): 228-37.

13. Shah RB, Zhou M. Recent advances in prostate cancer pathology: Gleason grading and beyond. Pathol Int 2016; 66(5): 260-72.

14. Hashmi AA, Khan EY, Irfan M, Ali R, Asif H, Naeem M, et al. ERG oncoprotein expression in prostatic acinar adenocarcinoma; clinicopathologic significance. BMC Res Notes 2019; 12(1): 35-38.

15. Abdelsalam RA, Khalifeh I, Box A, Kalantarian M, Ghosh S, Abou-Ouf $\mathrm{H}$, et al. Molecular characterization of prostate cancer in Middle Eastern population highlights differences with Western populations with prognostic implication. J Cancer Res Clin Oncol 2020; 146(7): 1701-09.

16. Verdu M, Trias I, Roman R, Rodon N, Garcia-Pelaez B, Calvo M, et al. ERG expression and prostatic adenocarcinoma. Virchows Arch 2013; 462(6): 639-44.

17. Dubovenko A, Serebryiskaya T, Nikolsky Y, Nikolskaya T, Perlina A, Je Bailey L, et al. Reconstitution of the ERG gene expression network reveals new biomarkers and therapeutic targets in ERG positive prostate tumors. J Cancer 2015; 6(6): 490-501.

18. Liu H, Shi J, Wilkerson M, Yang XJ, Lin F. Immunohistochemical evaluation of ERG expression in various benign and malignant tissues. Ann Clin Lab Sci 2013; 43(1): 3-9. 